\title{
REPORT OF A CASE OF WERNICKE'S SYNDROME COMPLICATING PREGNANCY AND ASSOCIATED WITH OCULAR COMPLICATIONS*
}

BY

\author{
George BLACK
}

LEEDS

WERNICKE first published an account of the condition in 1881 . He based his account upon three cases and described the condition as one of acute superior haemorrhagic polioencephalitis. Two of his cases were due to chronic alcoholism and the third to sulphuric acid poisoning. All the cases showed minute haemorrhagic foci situated in the walls of the third ventricle, the grey matter around the aqueduct and the floor of the fourth ventricle. Quite early, the association of polyneuritis and Korsakoff's syndrome with the special cerebral signs were noted. Wernicke considered that the condition was an acute inflammatory process. In recent years, however, the views regarding the aetiology and the pathology of the condition have changed. Neuberger emphasised the occurrence of the condition as a complication of a variety of primary diseases. Furthermore, the condition is no longer regarded as an encephalitis, but rather as an encephalopathy or encephalosis.

Campbell and Biggart published an excellent survey in the past few years of twelve cases which came to post-morten. Of these twelve cases, only one occurred in chronic alcoholism. There were three cases of gastric carcinoma and two of hyperemesis gravidarum. Other cases occurred in pernicious anaemia, chronic gastritis, bronchiectasis and pyosalpynx. They found focal lesions consisting of small petechial haemorrhages with vascular proliferation. There was an absence of inflammatory infiltration and only slight evidence of injury to nerve cells. The lesions had a selective distribution, the corpora mammillaria being the most characteristic site. In fact, histological examination of these bodies they regarded as essential to diagnosis. The lesions are almost always symmetrical involving the grey matter surrounding the third ventricle, the peri-aqueductal grey matter, hence involving the ocular nuclei, and the floor of the fourth ventricle. The lesions suggest a toxic agent, producing a loss of vascular tone and stasis.

The main clinical features of this disease are drowsiness, leading to coma, associated with eye signs : retinal haemorrhages of the type which I will describe later, nystagmus, ophthalmoplegia and

* Read before the North of England Ophthalmological Society on December 14, 1940, at Leeds. 
the Argyll Robertson pupil. In some cases described, haemorrhages occur in the optic nerves, producing central scotoma. It is to be noted that these signs often occur as a terminal event in some such serious condition as carcinoma of the stomach and are not always accurately observed or are regarded as features of the general cachexia.

An understanding of the aetiology of the disease depends upon the clinical picture, the pathological findings and also on experimental work. Wernicke's original view of some exogenous infection is not substantiated by the histological appearances. The lesions do not resemble infective forms of encephalitis. The two modern views are (1) that the condition is due to an endogenous toxin and (2) that a vitamin deficiency is responsible. Neuberger suggested that damage to the liver by such an agent as alcohol or by metastasis, allowed a toxin from the gut to circulate and this produced the cerebral changes. There is little experimental evidence to support this view and Campbell and Biggart did not find serious liver damage in their cases. On the other hand, quite impressive evidence has been amassed to show that Vitamin $B_{1}$ deficiency is largely responsible for the onset of Wernicke's disease. It has been noted that chronic alcoholism may produce both Wernicke's disease and polyneuritis. This polyneuritis is similar to that of beriberi, which is due to a $B_{1}$ deficiency. It has been shown that alcoholic polyneuritis rapidly improves under $B_{1}$ therapy, in spite of the continued use of alcohol, and, furthermore, that the alcoholic with a low intake of $B_{1}$ is the individual liable to polyneuritis. It is consistent to assume that the same deficiency may be responsible for the encephalopathy.

In cases of Wernicke's disease occurring in the course of pregnancy, of which my case is an example, persistent vomiting is a leading feature. This leads to a poor intake of food with a consequent deficiency of accessory factors. Many of the cases of Wernicke's disease have in common gastro-intestinal disturbance placing limits upon the diet and probably causing defective absorption.

I will refer briefly to the experimental production of the disease. Prickett showed that rats fed on a diet deficient in $B_{1}$ developed foci of congestion, of haemorrhage and parenchymatous degeneration in the pons and medulla. The most interesting work, however, has been done on pigeons by Alexander, who was able to reproduce the characteristic selective pathological signs of Wernicke's disease. Early last year, he published the following conclusions :

1. Wernicke's haemorrhagic polioencephalitis can be produced with regularity in pigeons deficient in Vitamin $B_{1}$, as a complication of beriberi after large supplies of other vitamins have been given. 
2. If pigeons are kept on an entirely vitamin-free diet, the resulting beriberi will only rarely be complicated by Wernicke's disease.

3. Wernicke's disease cannot be produced in pigeons receiving crystalline $B_{1}$, although they may be deprived of any other vitamins for a period of six months.

4. The observations suggest that Vitamin $\mathbf{B}_{1}$ possesses antiangiodegenerative properties in addition to anti-neuritic properties, and smaller amounts of Vitamin $B_{1}$ are sufficient to act as an anti-angiodegenerative agent than are necessary for anti-neuritic action.

5. The administration of high doses of Vitamins $A, B_{2}, C$, and $D$ in Vitamin $B_{1}$ deficiency probably raises the Vitamin $B_{1}$ requirements of tissues and angiodegeneration manifests itself soon after the onset of neuronal degeneration.

It must be stated that $B_{1}$ deficiency alone may not be responsible for Wernicke's encephalopathy, because this disease is not found in beriberi, which is probably the best example of $B_{1}$ avitaminosis. It is possible that the disease may be due to the combined effects of $B_{1}$ deficiency and an endogenous toxin.

My patient happily survived and I have no pathological confirmation of the disease, except the striking changes found in the fundi during the acute phase. No doubt, similar haemorrhages to those which I saw were present in the grey matter surrounding the third and fourth ventricles.

When three months pregnant, she began to get very severe headaches and sickness. The vomiting continued all day long and during the night. A month or six weeks later she began to notice severe failure of vision which led to her admission to hospital. I first saw her at this stage, when her right eye showed two or three large deeply-placed retinal haemorrhages. The left eye also showed several large retinal haemorrhages around the disc. There were, however, no exudates and generally the appearances were unlike those of albuminuric retinitis. The vision of each eye was reduced to hand movements. She had, unlike some cases reported, no ocular paralysis, but she was mentally very drowsy. I saw the case at the Maternity Hospital, where the combination of severe headache and retinal haemorrhages is almost invariably associated with pregnancy toxaemia and nephritis with a considerable rise in blood pressure. It is well known that pregnancy toxaemia, combined with eye signs, is often best treated by termination of the pregnancy. This case, however, differed from the majority in having a somewhat atypical fundus picture, a normal blood pressure and a clear urine. Nevertheless, on first seeing the severity of the fundus changes, I formed 
the impression that termination of the pregnancy would be necessary. However, on account of these unusual features and the existence of the cerebral signs, on the advice of my colleague, Dr. MacAdam, it was decided to treat her intensively with the Vitamin B complex. The results were dramatic. She was given daily $20 \mathrm{mg}$. of Vitamin $B_{1}$ by injection and three $50 \mathrm{mg}$. tablets of nicotinic acid by mouth. The subjective response was remarkable. After one injection and two tablets, her vision was much improved and within four hours she said that she was able to tell the time by the clock in the ward. There was no visible change in her fundi during the first few hours of treatment to explain the dramatic improvement in vision. This indicates that the haemorrhages were not the only or even chief cause of the visual defect, but rather the associates of a toxic or nutritional effect upon the nerve elements. Treatment of the avitaminosis showed its anti-neuritic effects first in improvement of vision and its antiangiodegenerative effect later. She rapidly improved and was able to read the paper within a fortnight. She left the hospital and later had an uncomplicated confinement and normal puerperium. To-day she has vision of $6 / 6$ unaided in both eyes and only slight signs are visible, limited to temporal pallor of the right disc.

The case was probably not of severe degree at the time treatment was started. The absence of ophthalmoplegia suggests that the cerebral changes were not extensive. I believe the fundus picture of large darkish haemorrhages splashed around the disc to be probably fairly characteristic of the condition. These fundus lesions illustrate the close correspondence between the retina and brain. I think that her condition sufficiently resembled the published accounts of Wernicke's disease as to be so regarded and, if she was such a case, she is further support for the view that this condition is due in part at least to deficiency of Vitamin $\mathbf{B}_{1}$.

I wish to thank Mr. Andrew Claye for the opportunity of examining this case.

\section{REFERENCES}

Campbell and Biggart.-Jl. of Path. and Bact., Vol. XLVIII, No. 2.

PRICKetT, C. C.-Amer. Jl. of Physiol., Vol. CVII, p. 459.

Alexander, L.-Amer. Jl. of Path., January, 1940. 